\title{
La Conférence de l'Entente balkanique à Bucarest (20-22 février 1939) : le chant du cygne d'une tentative de confédération
}

The Balkan Pact's Conference in Bucharest: the Twilight of a Federation Attempt

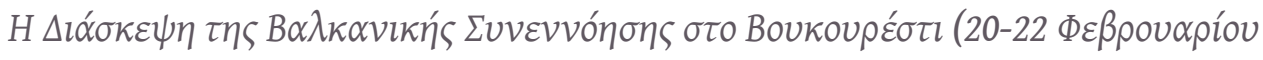

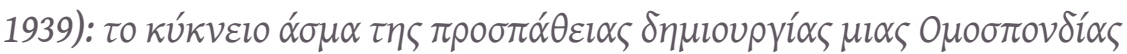

\section{Alkisti Sofou}

\section{(2) OpenEdition}

\section{Journals}

Édition électronique

URL : https://journals.openedition.org/ceb/4907

DOI : $10.4000 /$ ceb.4907

ISSN : 2261-4184

Éditeur

INALCO

Référence électronique

Alkisti Sofou, «La Conférence de l'Entente balkanique à Bucarest (20-22 février 1939) : le chant du cygne d'une tentative de confédération », Cahiers balkaniques [En ligne], 42 | 2014, mis en ligne le 28 mai 2014, consulté le 07 juillet 2021. URL : http://journals.openedition.org/ceb/4907 ; DOI : https:// doi.org/10.4000/ceb.4907

Ce document a été généré automatiquement le 7 juillet 2021.

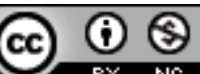

Cahiers balkaniques est mis à disposition selon les termes de la Licence Creative Commons Attribution - Pas d'Utilisation Commerciale 4.0 International. 


\title{
La Conférence de l'Entente balkanique à Bucarest (20-22 février 1939) : le chant du cygne d'une tentative de confédération
}

\author{
The Balkan Pact's Conference in Bucharest: the Twilight of a Federation Attempt

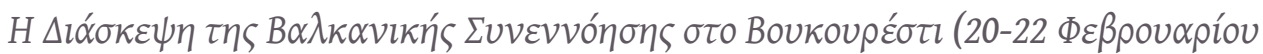

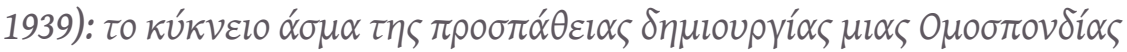

\section{Alkisti Sofou}

\section{Introduction}

1 L'Entente balkanique, créée en février 1934 entre la Turquie, la Yougoslavie, la Roumanie et la Grèce, avait comme objectif la défense de ses États membres contre le danger du révisionnisme bulgare ${ }^{1}$. Fruit de l'initiative du ministre des Affaires étrangères de Roumanie Nicolae Titulesco, l'Entente balkanique exprimait aussi le désir de préserver ses États membres de l'Allemagne hitlérienne qui semblait avoir repris son traditionnel Drang nach Osten ainsi que de la politique maximaliste de Mussolini et ses visions vers l'Est.

2 L'Entente balkanique suscite peu d'intérêt auprès des historiens. Cependant à l'époque qui nous intéresse, elle suscita un intérêt bien supérieur auprès de la diplomatie des puissances européennes. Et pour cause: dans une Europe centrale et orientale redessinée par les traités annexes au traité de Versailles, l'ordre balkanique s'est construit en relation avec celui de l'Europe. Pour suivre l'évolution de l'Entente balkanique, ses frictions et ses fractures, nous nous appuierons sur les discours prononcés lors de la session de son Conseil permanent. Nous évoquerons aussi des articles de la presse grecque, roumaine, turque et française ainsi que des documents diplomatiques. Ceci nous conduira à tracer les grandes lignes des différents aspects des 
relations interbalkaniques et des enjeux de la politique des puissances européennes. En intégrant donc l'Entente balkanique dans le cadre politique européen, nous tenterons de rendre compte des raisons de sa dissolution.

\section{Le conseil de février 1939 à Bucarest}

3 Le Conseil permanent de l'Entente balkanique s'est réuni à Bucarest du 20 au 22 février 1939, sous la présidence de Grégoire Gafenco, ministre des Affaires étrangères de Roumanie. La Grèce était représentée par le dictateur Ioannis Metaxás, Président du Conseil de l'Entente, Premier ministre et ministre des Affaires étrangères de Grèce, la Turquie et la Yougoslavie étant représentées par leurs ministres des Affaires étrangères, Saraçoglou et Tsintsar-Marcovitch.

4 L'esprit régnant est éloquemment évoqué par le ministre des Affaires étrangères de Roumanie, Grégoire Gafenco, dans son article intitulé «La politique étrangère de la Roumanie », paru dans la revue diplomatique française Ambassades et Consulats : "La Roumanie, écrit-il, persiste dans sa volonté de collaboration dans tous les domaines, avec son alliée la Yougoslavie, à laquelle la lient des intérêts communs dans les Balkans ». Il continue en écrivant que son pays "s'appuie en toute confiance sur le pacte balkanique, car celui-ci le rapproche et le relie à deux autres puissances, la Turquie et la Grèce, qui partagent ses vues et ses décisions concernant la consolidation de l'état actuel et la défense de l'ordre établi dans la région du Sud-Est européen ${ }^{2}$ ».

5 Malgré l'apparente solidarité, à partir de 1937, au sein de l'Entente, il y a divergence d'opinions : la Turquie et la Grèce voulaient limiter les responsabilités de l'Entente à la région balkanique. La Yougoslavie limitait son rôle à une présence nominale aux débats et aux décisions du Conseil Permanent. La Roumanie voulait rattacher l'Entente aux alliances «protégées» par la France. Cette divergence se traduit par des accords unilatéraux qui sapent l'alliance.

6 Le 29 janvier 1937, la Yougoslavie a signé un pacte avec la Bulgarie sans prévenir ses partenaires ${ }^{3}$ tandis que la Roumanie, la Grèce et la Turquie observaient avec inquiétude ce rapprochement. Le gouvernement yougoslave a tenté d'apaiser leurs inquiétudes en déclarant que les accords signés avec la Bulgarie ne pourraient en aucun cas compromettre l'accord de son pays avec les autres membres de l'Entente ${ }^{4}$. Néanmoins, le 25 mars de la même année la Yougoslavie signa un autre pacte avec l'Italie. Cette initiative unilatérale fissura l'union de l'Entente et porta atteinte aux intérêts de la Grèce. Le pacte italo-yougoslave révoquait le traité d'amitié signé le 23 septembre 1928 entre Mussolini et Venizélos, selon lequel l'Italie empêcherait toute revendication yougoslave sur Thessalonique ${ }^{5}$.

7 Un mois plus tard, Ioannis Metaxás accepta de signer malgré les réticences, voire les protestations de son gouvernement et de l'État-major grec, l'accord militaire de l'Entente balkanique. Les protestations étaient motivées par l'article 14 dudit accord qui reconnaissait le droit à la Yougoslavie de se servir du port de Thessalonique en cas de conflit avec l'Italie.

8 En 1938, Hitler profite de «la politique d'apaisement» du Foreign Office ainsi que des hésitations du Quai d'Orsay et ne cache plus ses ambitions. Après l'annexion de l'Autriche au Reich, l'étau se resserre autour de la Tchécoslovaquie, et la Roumanie est menacée. Les pays membres de l'Entente veulent renforcer leur alliance pour mieux se 
défendre contre l'expansionnisme germanique. Le journal turc Cumhuriyet se demande pourquoi « les États membres de l'Entente balkanique ne s'uniraient pas dans la défense contre toute probabilité d'agression de leurs frontières nationales [...]; les portes de l'Entente sont toujours largement ouvertes, écrit-il, même pour la Bulgarie ${ }^{6}$ ». À l'instigation de l'Angleterre, ils entament des négociations avec la Bulgarie en vue d'une adhésion.

\section{Metaxás et la recherche de la participation bulgare}

C'est ainsi que le 31 juillet 1938, à Thessalonique, Metaxás, alors Président du Conseil des ministres de l'Entente balkanique, accepta de signer avec le Premier ministre de la Bulgarie, Kiosséivanoff, un pacte qui révoquait les dispositions du traité de Neuilly et de Lausanne concernant les zones démilitarisées. En somme, les membres de l'Entente permettaient à la Bulgarie de s'équiper en armement. Le premier ministre bulgare, désireux de clarifier la position de son pays aux yeux de son opinion publique, déclara que la signature de l'accord ne signifiait pas impérativement que la Bulgarie garantirait le statu quo établi par le traité de Sèvres.

10 Malgré cette déclaration, l'accord de Thessalonique a été ratifié lors du Conseil permanent de l'Entente qui s'est réuni à Bucarest du 20 au 22 février 1939.

11 Grégoire Gafenco, lors du dîner organisé en l'honneur des délégations, prononça un discours pour exalter la volonté de paix de l'Entente. Le passage le plus remarqué est le suivant : « Nous devons à Monsieur Metaxás d'avoir, l'année passée, signé en notre nom à Salonique un accord avec la Bulgarie. Cette importante réalisation témoigne de notre désir de convier sur un pied de parfaite égalité tous les peuples voisins à se joindre à nous pour développer en commun des rapports d'amitié et de bon voisinage $\mathrm{e}^{7}$ ».

Ioannis Metaxás, répondant à Gafenco, dit entre autres : «Est-il nécessaire de répéter que l'Entente balkanique ne poursuit aucun but agressif? Qu'elle est ouverte à toutes les bonnes volontés ainsi qu'à toute collaboration sincère? Le récent accord de Thessalonique, librement négocié et conclu entre les États de l'Entente et la Bulgarie, est l'aboutissement heureux de négociations empreintes d'une bonne volonté réciproque en vue de créer une atmosphère de détente et d'entente entre les États de l'Entente balkanique et la Bulgarie permettant le développement des plus cordiales relations ${ }^{8} »$.

13 Il est assez étonnant de voir les dirigeants balkaniques, dans une Europe où la sécurité collective devient de plus en plus illusoire, s'engager dans une politique bipolaire voire schizophrène. En même temps qu'ils sollicitaient l'aide et le soutien de la France et de la Grande-Bretagne, ils consacraient une grande partie de leur effort à consolider et à faire vivre l'Entente balkanique complètement dépassée par les évènements, en fixant des objectifs totalement antinomiques avec ceux d'autres pays membres ou ceux de leurs puissances protectrices.

Pour mieux comprendre cette "dualité » nous devons faire appel aux protagonistes. Grégoire Gafenco écrit dans ses mémoires: "Il fallait concilier l'idée de sécurité balkanique, qui ne pouvait se passer de l'appui indirect que lui donnait la politique de garantie de l'Angleterre et de la France, et l'idée d'indépendance balkanique, qu'il était indispensable de pouvoir opposer à l'Allemagne, pour désarmer sa méfiance et lui enlever tout prétexte d'intervention ${ }^{9}$ ». Nicolas Politis, un des diplomates balkaniques 
les plus expérimentés de sa génération, ministre de Grèce en France, est sur la même longueur d'onde. Dans une note qu'il envoie en 1940 on peut lire : " Malgré le profond respect et l'amour sincère que je nourris pour la France et la Grande-Bretagne, ma confiance en leur jugement politique est plus que réduite quand il s'agit du sort de notre patrie. [...]. Le seul contrepoids efficace, certain et permanent est une collaboration étroite entre les pays des Balkans. Les pays des Balkans n'ont aucun intérêt à rechercher l'intervention d'une quelconque grande puissance dans leurs relations ${ }^{10} »$.

Les deux hommes évoquent les objectifs primordiaux actuels : leur sécurité et leur indépendance à l'égard " de l'égoïsme naturel des grandes puissances ", pour reprendre les termes de Nicolas Politis.

On retrouve dans le quotidien francophone turc La République ${ }^{11}$ un écho de cette interprétation:»Les petits États pris isolément peuvent ne pas avoir une grande signification. Mais lorsque quatre ou cinq d'entre eux s'allient pour soutenir la paix, on les voit former une grande puissance digne d'être accueillie avec considération. »

Le 22 février, à l'issue de la conférence, Metaxás exprime sa satisfaction lors de la conférence de presse : «Selon le vieil adage, Tout est bien qui finit bien. Cependant, pour notre conférence on devrait dire Tout est bien qui commence bien. Depuis notre arrivée dans la splendide capitale de la Roumanie, l'ambiance de notre conférence était belle comme ce temps radieux ${ }^{12} »$.

Pendant que l'allégresse est générale, les nuages d'orage approchent. Le 15 mars 1939, les troupes allemandes envahissent la Bohême et la Moravie. Quatre jours plus tard, la Roumanie rejette les propositions soviétiques (19 mars) d'un accord d'assistance mutuelle. À l'inverse, elle se rapproche de l'Allemagne, à l'initiative du roi, dans la perspective d'échanges économiques (selon le système du clearing, c'est-à-dire d'un troc en nature) comparable à ceux liant le Reich à des pays comme la Hongrie ou la Bulgarie. En avril, l'Italie envahit l'Albanie et la rattache à la couronne. Le 13 avril, Édouard Daladier et Neville Chamberlain annoncent de façon platonique l'assistance promise à la Grèce et à la Roumanie. Le pacte germano-soviétique, signé le 23 août 1939, sème la panique dans les Balkans et les grandes puissances commencent à intervenir directement dans les affaires des États balkaniques. L'Entente n'ayant pas réussi à les tenir à l'écart du conflit, ceux-ci l'abandonnent l'un après l'autre. La conférence de Belgrade de février 1940 fut l'ultime rencontre.

\section{Conclusion}

19 L'Entente balkanique pourrait être incluse dans les alliances régionales signées en 1921-1922 dans le but de maintenir la situation telle qu'elle était définie par le traité de Paris $^{13}$. Les États balkaniques répondirent aux impératifs du moment et assurèrent un équilibre - dynamique, mais précaire - qui permit leur consolidation, voire leur émancipation. 


\section{BIBLIOGRAPHIE}

Ambassades et Consulats, $13^{\mathrm{e}}$ année, janvier-février, Paris, 1939.

Ministère (français) des Affaires étrangères, Bulletin périodique de la presse roumaine, 95 , $1^{\mathrm{er}}$ octobre-8 novembre 1930.

Ministère (français) des Affaires étrangères, Bulletin périodique de la presse roumaine, 147, 14 décembre-22 février, 1939.

Ministère (français) des Affaires étrangères, Bulletin périodique de la presse yougoslave, 90 , 16 avril-15 juin 1930.

Correspondance de l'Orient, $\mathrm{n}^{\circ}$ 494, Paris, 1939.

Gafenco, Grégoire (1944), Préliminaires de la guerre à l'Est. De l'accord de Moscou aux hostilités de Russie, Paris.

Kathimerini, 21 février 1939.

Kitsiki, Dimitri, (1969), « Les projets de l'Entente balkanique », Revue historique, t. CCXLI, 1969, p. 123-130.

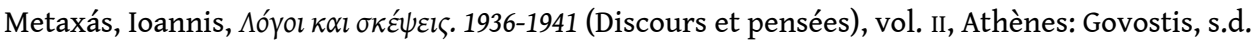

Oşca, Alexandru, (2008) "Romania and the Balkan Pact (1930-1934). A short historiography study and general considerings”, Codrul Cosminului, 14, Suceava, 2008, pp. 271-282.

Prévélakis, Constantin, (2008), « Et s'il fallait revenir sur l'Entente balkanique ? La construction manquée d'un ordre régional en Europe du Sud-Est à la fin des années 1930 ", Francia, 35, 2008, p. 317-326.

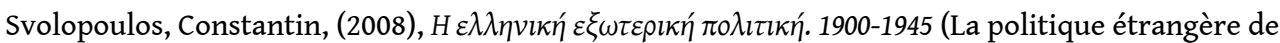
la Grèce), Athènes: Estia.

\section{NOTES}

1. Elefthérios Venizélos désapprouve la création de l'Entente balkanique et soutient que les accords unilatéraux que la Grèce avait déjà signés avec la Turquie et l'Italie garantissaient l'intégrité territoriale de la Grèce. Il exposa ses arguments dans une série d'articles publiés dans

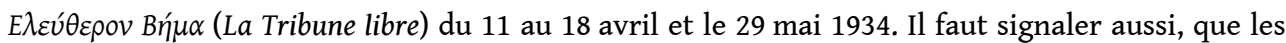
partis communistes des Balkans se sont opposés à toute tentative d'union ou de confédération. Leur attitude pourrait être résumée dans le principe "Les Balkans aux peuples balkaniques". Pour plus d'informations, voir : Bulletin périodique de la presse yougoslave, 90, 16 avril-15 juin 1930, p. 5 et Bulletin périodique de la presse roumaine, 95, $1^{\mathrm{er}}$ octobre-8 novembre 1930, p. 2.

2. Ambassades et Consulats, $13^{\mathrm{e}}$ année, janvier-février, Paris, 1939, p. 14.

3. Pour plus d'informations sur le rapprochement bulgaro-yougoslave, voir : Kitsikis, Dimitris, «Les projets de l'Entente balkanique », Revue historique, t. CCXLI, 1969, p. 123-130.

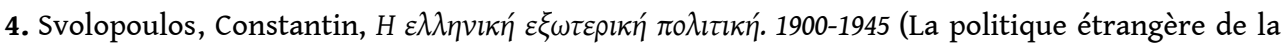
Grèce), Estia, Athènes, 2008, p. 249-250.

5. Pour plus d'informations, voir : Oșca, Alexandru, « Romania and the Balkan Pact (1930-1934). A short historiography study and general considerings", Codrul Cosminului, 14, Suceava, 2008, pp. 271-282. 
6. Correspondance de l'Orient, $\mathrm{n}^{\circ} 494$, Paris, 1939 , p. 91.

7. Bulletin périodique de la presse roumaine, 147, 14 déc.-22 fév. 1939, p. 5.

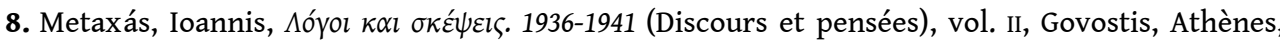
s.d., p. 28 - Cf. Bulletin Périodique de la presse roumaine, 147, 14 déc.-22 fév. 1939, p. 5 et Kathimerini, 21 fév. 1939, p. 1.

9. Grégoire Gafenco, Préliminaires de la guerre à l'Est. De l'accord de Moscou aux hostilités de Russie, Paris, 1944, p. 296.

10. Cité dans: Prévélakis, Constantin, «Et s'il fallait revenir sur l'Entente balkanique? La construction manquée d'un ordre régional en Europe du Sud-Est à la fin des années 1930 ", Francia, 35, 2008, p. 321.

11. 25 février 1938.

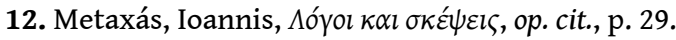

13. Kitsikis, Dimitris, « Les projets de l'Entente balkanique », op. cit.

\section{RÉSUMÉS}

L'Entente balkanique, créée en février 1934 entre la Turquie, la Yougoslavie, la Roumanie et la Grèce, avait comme objectif la défense de ses États membres contre le danger du révisionnisme bulgare. Fruit de l'initiative du ministre des Affaires étrangères de Roumanie, Nicolae Titulesco, l'Entente balkanique exprimait aussi le désir de préserver ses États membres de la poussée vers l'est de l'Allemagne hitlérienne et de la politique maximaliste de Mussolini.

Le Conseil permanent de l'Entente balkanique qui s'est réuni à Bucarest du 20 au 22 février 1939 fut l'avant-dernier. En intégrant l'Entente balkanique dans le cadre politique européen, nous tenterons de suivre son évolution, ses frictions et ses fractures, afin de rendre compte des raisons de sa dissolution. Nous tracerons donc les grandes lignes des différents aspects des relations interbalkaniques et des enjeux de la politique des puissances européennes. Pour ce faire, nous nous appuierons sur les mémoires du ministre des Affaires étrangères de Roumanie, Grigore Gafencu, et sur les discours de Ioannis Metaxás prononcés lors de la session du Conseil permanent. Nous évoquerons aussi des articles de la presse grecque, roumaine et française ainsi que des documents diplomatiques.

The Balkan Entente also called Balkan Pact was signed in Athens (February 9, 1934) between Rumania, Greece, Yugoslavia and Turkey.

The member States aimed at defending their territorial status quo defined by the peace Treaty of Paris against attack by Bulgaria or Albania. They also aspired to strengthen their security towards the Italian maximalism and the hitlerian eastward expansion.

The Conference in Bucharest is the second last. The Balkan Pact became defunct in 1940. Here we propose to examine the reasons of its dissolution. We shall focus on the aspects of the interbalkan relations and the parameters of British and French foreign policy. Thus, we will rehearse articles of the Greek, Rumanian, French and Turkish press as well as the testimonies of the two protagonists: Grigore Gafencu and Ioannis Metaxas.

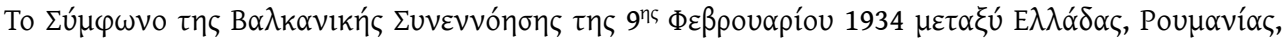

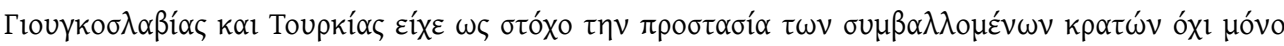

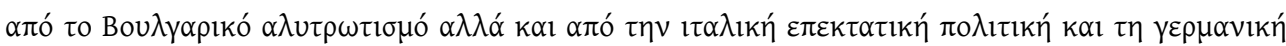


$\varepsilon \pi \varepsilon ́ \lambda \alpha \sigma \eta$.

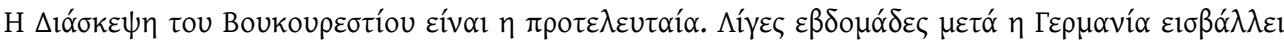

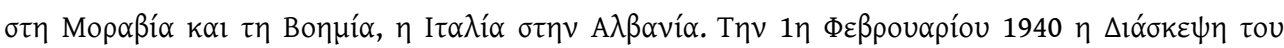

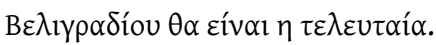

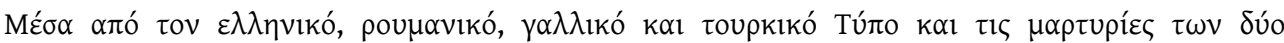

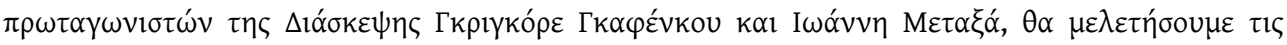

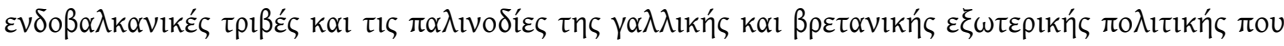

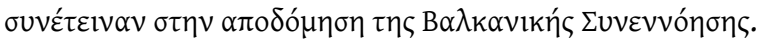

\section{INDEX}

motsclestr Balkan Antant1, Metaxas Ioannis (1871-1941), Titulesco Nicolae (1882-1941), Yunanistan, Romanya, Yugoslavya, Türkiye, Bulgaristan, Balkanlar, Yirminci yüzyılın ilk yarısı, Tarih, Diplomasi Tarihi

Keywords : Balkan Pact, Metaxas (1871-1941), Titulesco Nicolae (1882-1941), Greece, Romania, Yugoslavia, Turkey, Bulgaria, Balkans, Twentieth century, History, Diplomatic history

Mots-clés : Entente balkanique, entente balkanique, Metaxás Ioannis (1871-1941), Titulescu Nicolae (1882-1941), Titulesco Nicolae (1882-1941)

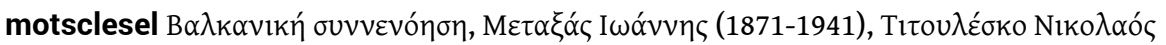

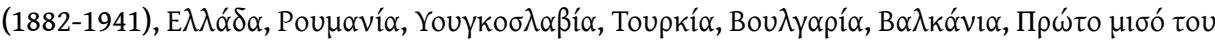

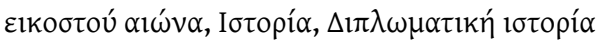

Thèmes : Histoire, Histoire diplomatique

motsclesmk БАЛКАН АНТАНТАТА, МЕТАКСАС ЈОАНИС (1871-1941), Titulesco НИКОЛАЕ

(1882-1941), ГРЦИЈА, РОМАНИЈА, ЈУГОСЛАВИЈА, ТУРЦИЈА, БУГАРИЈА, БАЛКАН, ПРВАТА ПОЛОВИНА НА ДВАЕСЕТТИОТ ВЕК. ИСТОРИЈА, ДИПЛОМАТСКА ИСТОРИЈА

Index géographique : Grèce, Roumanie, Yougoslavie, Turquie, Bulgarie, Balkans

Index chronologique : vingtième siècle -- début

\section{AUTEUR}

\section{ALKISTI SOFOU}

MCF

Université Paris IV) 